Supporting information for

\title{
Highly stable acid-induced emission-enhancing Cd-MOFs: Synthesis, characterization, and detection of glutamic acid in water and iron ions
}

\section{in acid}

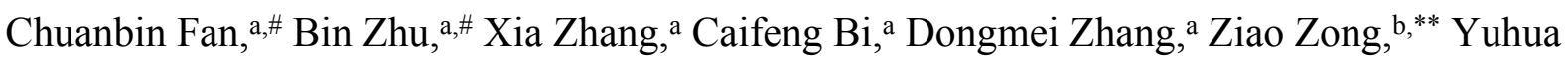
Fan $^{\mathrm{a}, *}$

a. College of Chemistry and Chemical Engineering, Ocean University of China, Qingdao, Shandong 266100, P.R. China

b. School of Laboratory Medicine, Youjiang Medical University for Nationalities, Baise, 533000 Guangxi, China

[\#] These authors have made equal contribution to this work.

*Corresponding author: fanyuhua301@163.com.

**Corresponding author: zongziao@126.com.

\section{Instruments and characterizations}

The Fourier-transform infrared (FT-IR) spectrum $\left(4000-400 \mathrm{~cm}^{-1}\right)$ was recorded with $\mathrm{KBr}$ pellets on the Nicolet 170SX spectrometer. X-ray single-crystal diffractometer with Mo-K $\alpha$ radiation was performed. Elemental analysis $(\mathrm{C}, \mathrm{H}$, and $\mathrm{N}$ ) was performed in a model 2400 Perkin-Elmer analyzer (EDX). Thermogravimetric (TG) analyses were performed on a NETZSCH TG 209F3 thermogravimetric analyzer with a ramp rate of $5{ }^{\circ} \mathrm{C} \cdot \mathrm{min}^{-1}$ from 30 to $700{ }^{\circ} \mathrm{C}$ under nitrogen conditions. The powder X-ray diffraction (PXRD) was collected on an Rigaku Ultima IV with a scan speed of $2^{\circ} \cdot \mathrm{min}^{-1}$ from 5 to $50^{\circ}$. Luminescent spectra were collected on a Hitachi FL-7000 spectrophotometer. X-ray photoelectron spectroscopy (XPS) were performed on Thermo ESCALAB XI+. The UV-vis absorption spectra for the dye adsorption performance were recorded in application of a Shimadzu UV-2550 UV-vis spectrophotometer. The porosities and BET surface areas of the samples were recorded by $\mathrm{N}_{2}$ adsorption/desorption isotherms (Micromeritics ASAP 2020 surface area and porosity analyzer). The scanning electron microscopy (SEM) were done on JEOL JSM-7600F. Fluorescence lifetime decay curves were measured by Edinburgh FLS980.

\section{X-ray crystallography}

The single crystals of Cd-MOFs 1-2 were treated and then suitable dimensions acquired crystals under an optical microscope. They were coated with high vacuum grease (Dow Corning Corporation) quickly, and mounted on glass fiber for data collection directly. We gathered X-ray crystallography data of 1-2 at 220/150 K on Bruker APEX-II CCD or Bruker SMART diffractometer, respectively, with Mo- $K \alpha$ radiation $(\lambda=0.71073 \AA)$ using the $\omega-2 \theta$ 
scan mode. The solution and refinement of the structure of 1-2 used ShelXT and ShelXL (Sheldrick, 2015) by direct methods. ${ }^{[1]}$ There were non-hydrogen atoms being defined by the Fourier synthesis method. Thermal and positional parameters were refined by the full matrix least-squares method (on $F^{2}$ ) to convergence. ${ }^{[2]}$ Hydrogen atoms were placed at the calculated positions and included as riding atoms with isotropic displacement parameters 1.2-1.5 times $U_{\text {eq }}$ of the attached $\mathrm{C}$ atoms. Their structures were studied through the Addsym subroutine of PLATON to assure that no additional symmetry could be applied to the models. ${ }^{[3]}$ The PLATON/SQUEEZE was employed to calculate the diffraction contribution of the solvent molecules and, thereby, to produce a set of solvent-free diffraction intensities. ${ }^{[4-8]}$
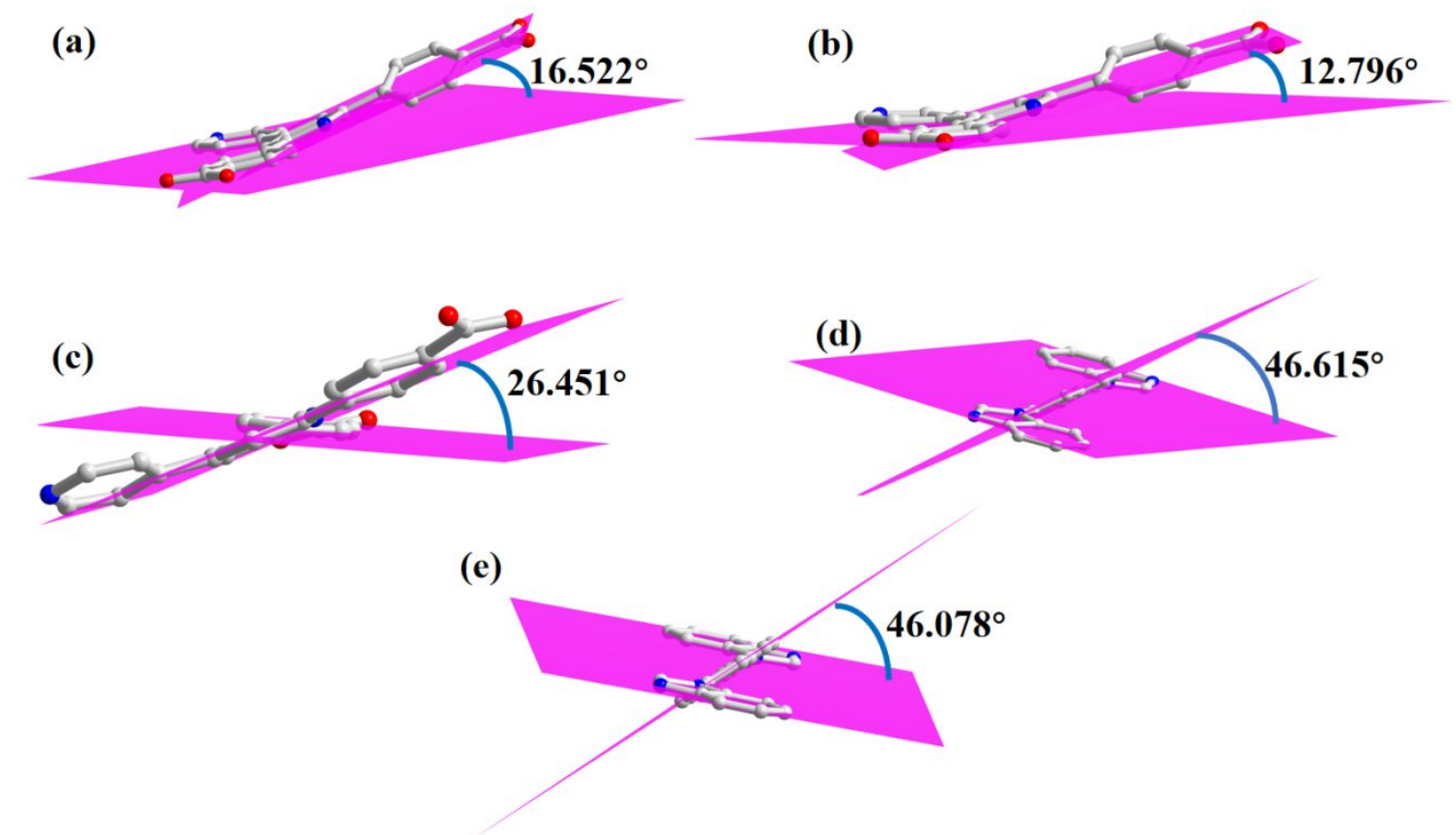

Figure S1. Dihedral angle of (a) pyridine and pyridine, (b-c) pyridine and benzene rings in bpydb ligand;(d-e) Dihedral angle of imidazoles and benzene ring in bbibp in Cd-MOF $\mathbf{1}$.

(a)

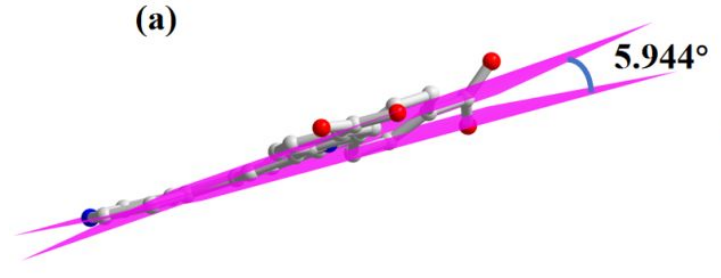

(c)

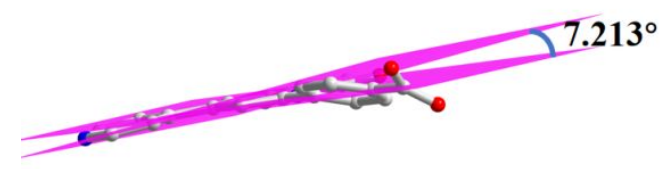

(b)

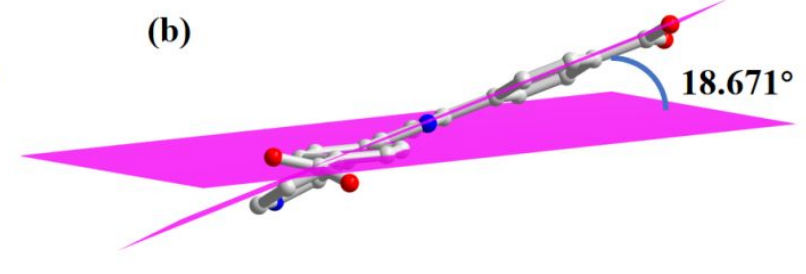

(d)

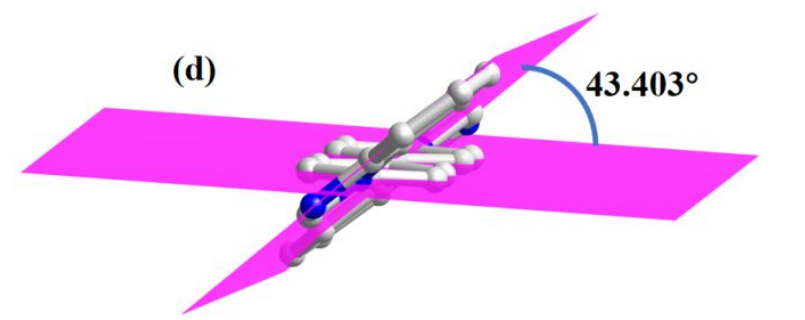

Figure S2. Dihedral angle of (a) pyridine and pyridine, (b-c) pyridine and benzene rings in bpydb ligand;(d) Dihedral angle of imidazoles and benzene ring in bbibp in Cd-MOF 2. 

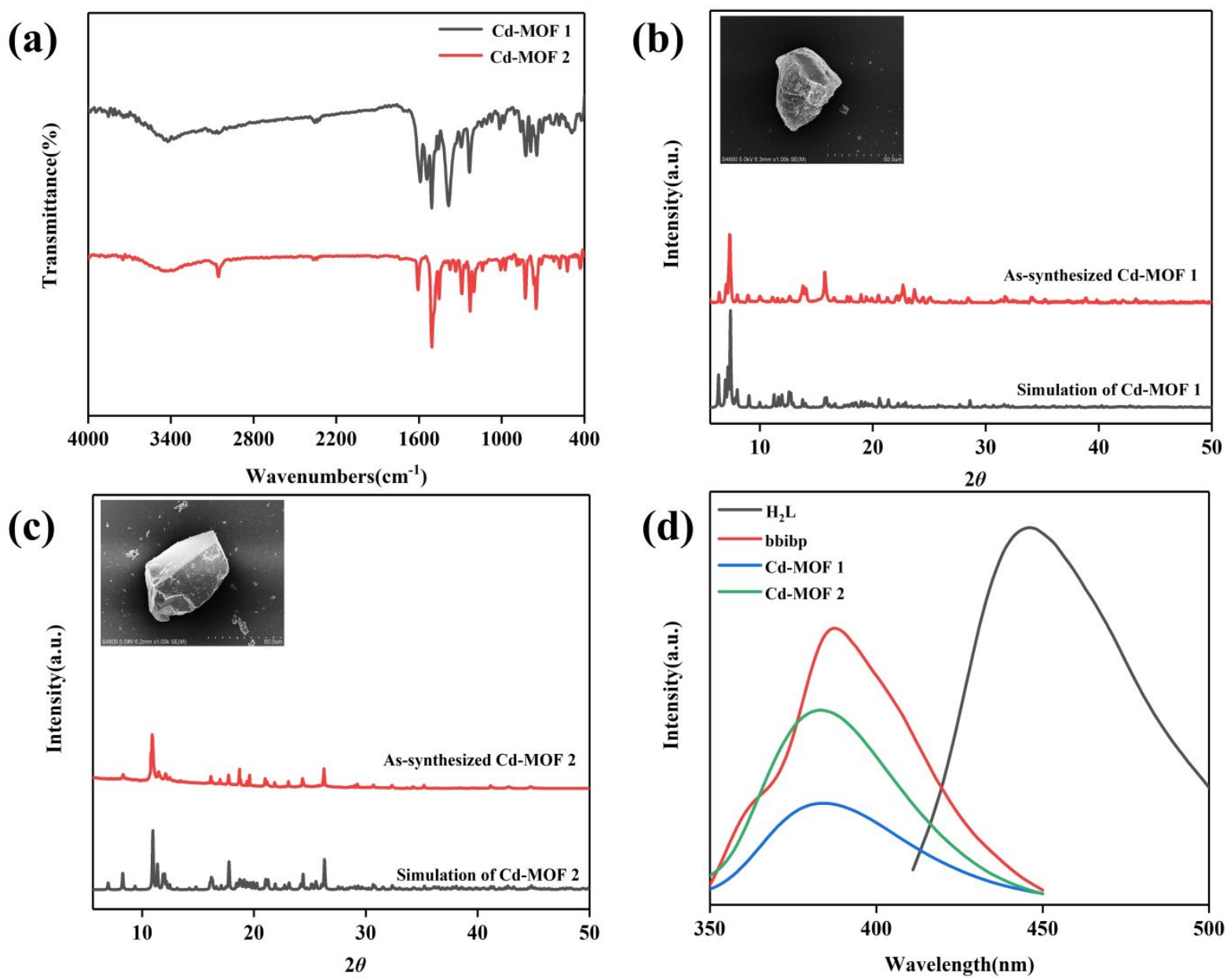

Figure S3. (a) The FT-IR spectra of Cd-MOFs 1-2; (b-c) The PXRD patterns of 1-2 (insert: SEM); (d) The solid-state fluorescence spectra of solid samples of 1-2 and ligands.
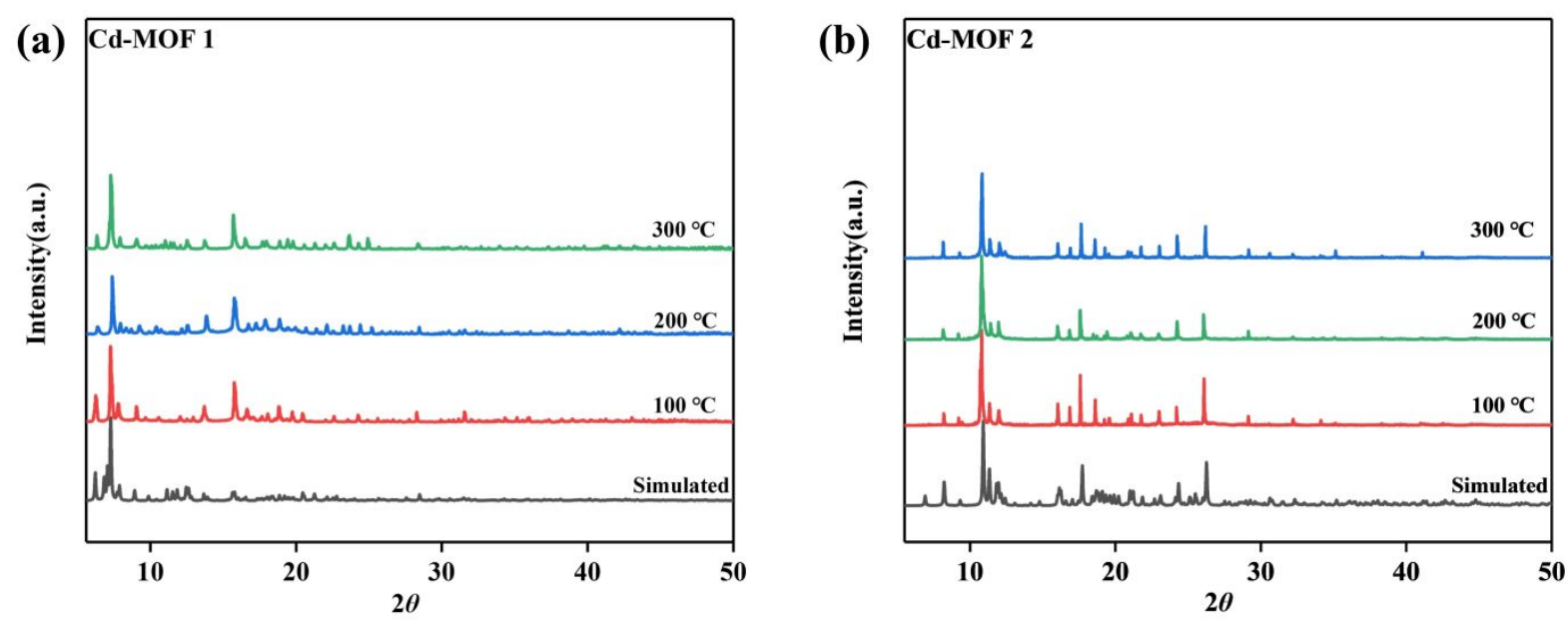

Figure S4. (a)-(b) The PXRD patterns of 1-2 under different temperatures treatment for one hour. 
(a)

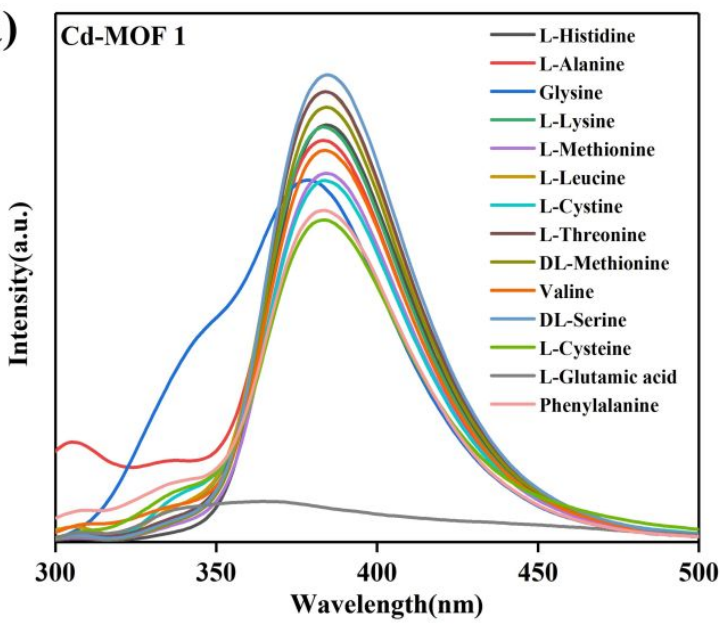

(b)

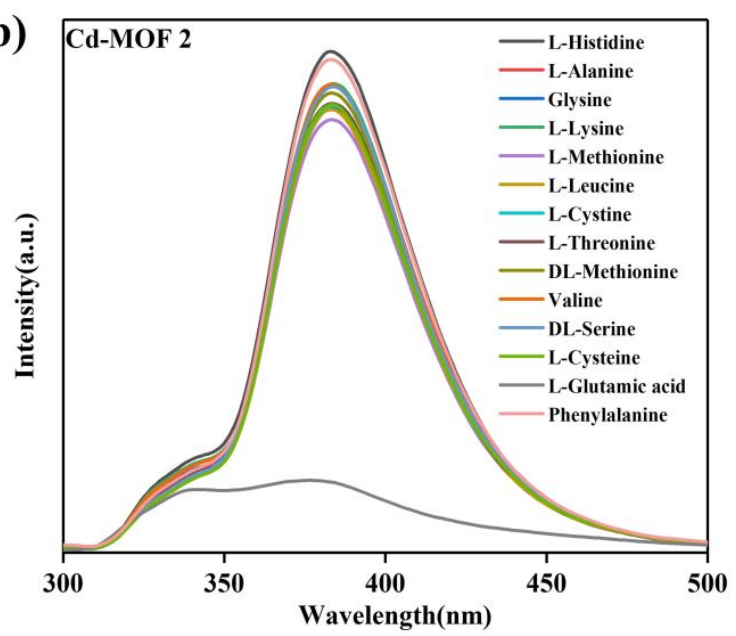

Figure S5. The fluorescent intensity of Cd-MOFs 1-2 for different amino acids.
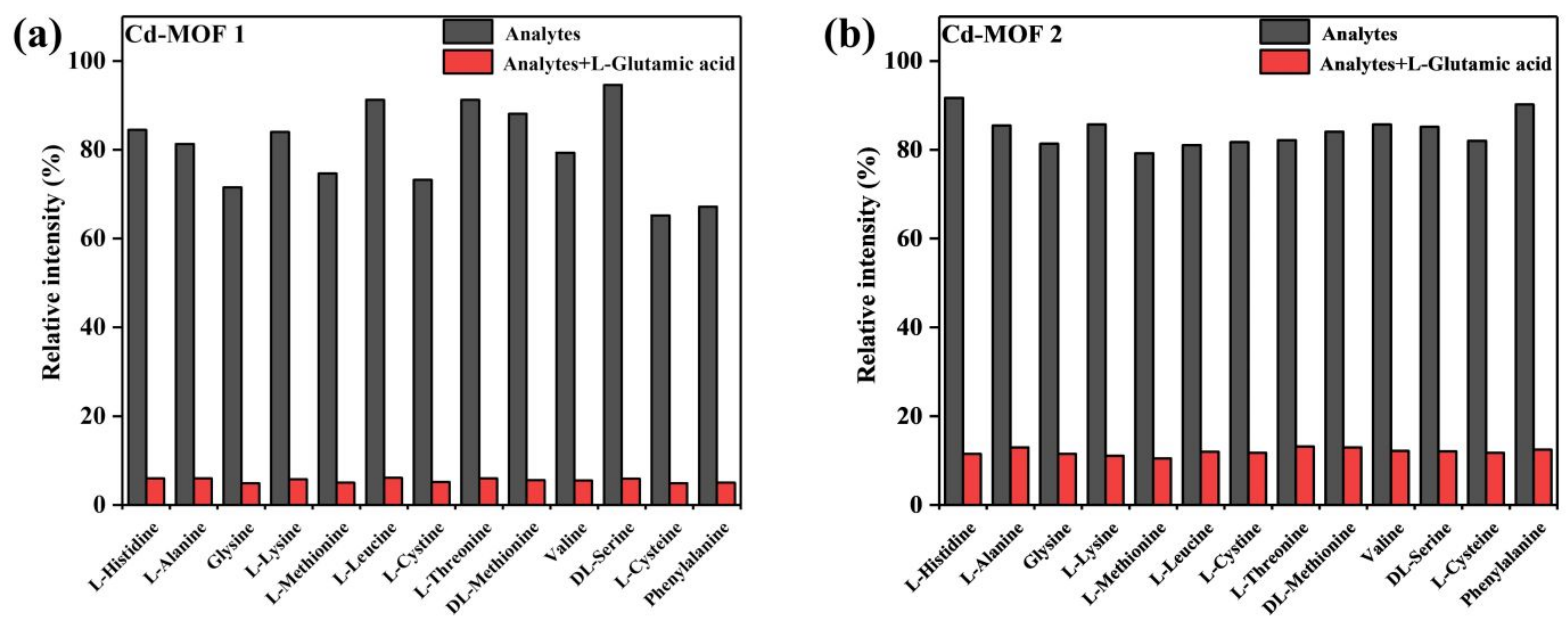

Figure S6. The relative luminescence intensities of Cd-MOFs 1-2 by different amino acids and selective detection of L-Glutamic acid in water.
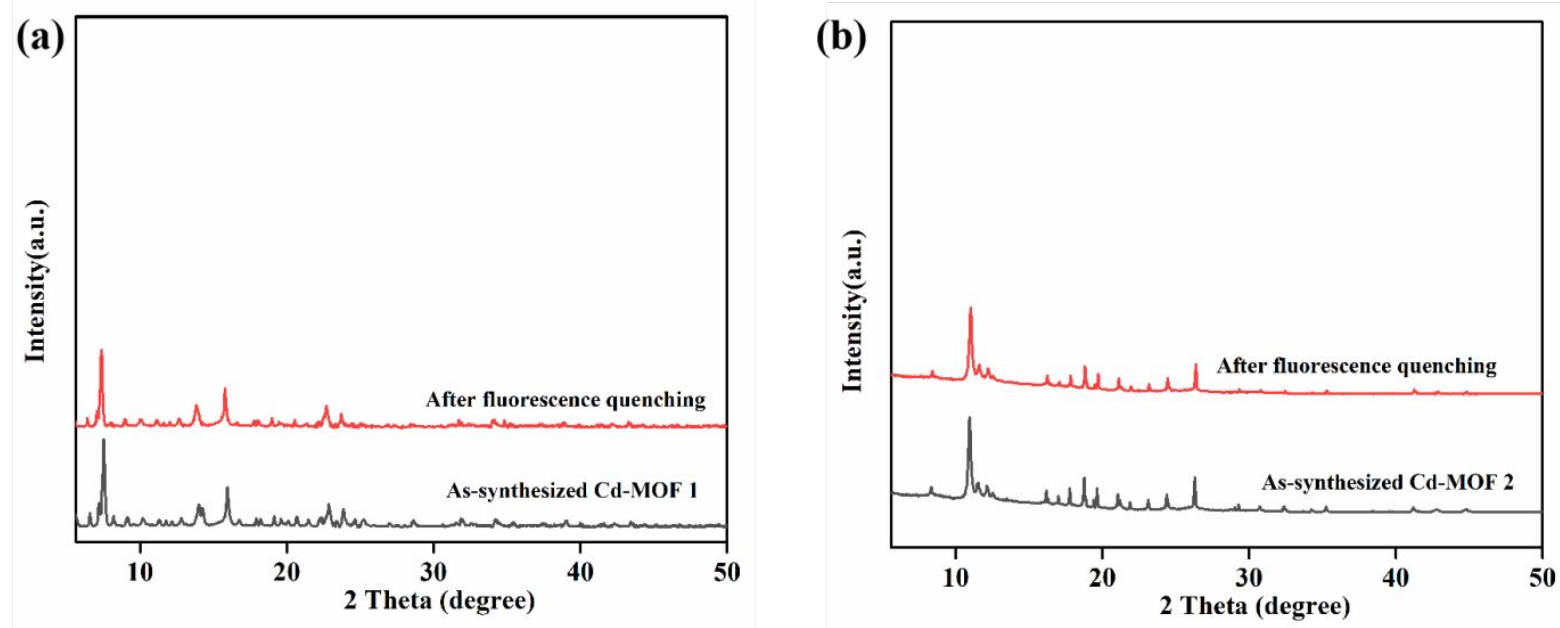

Figure S7. (a)-(b) The PXRD of Cd-MOFs 1-2 before and after fluorescence quenching. 


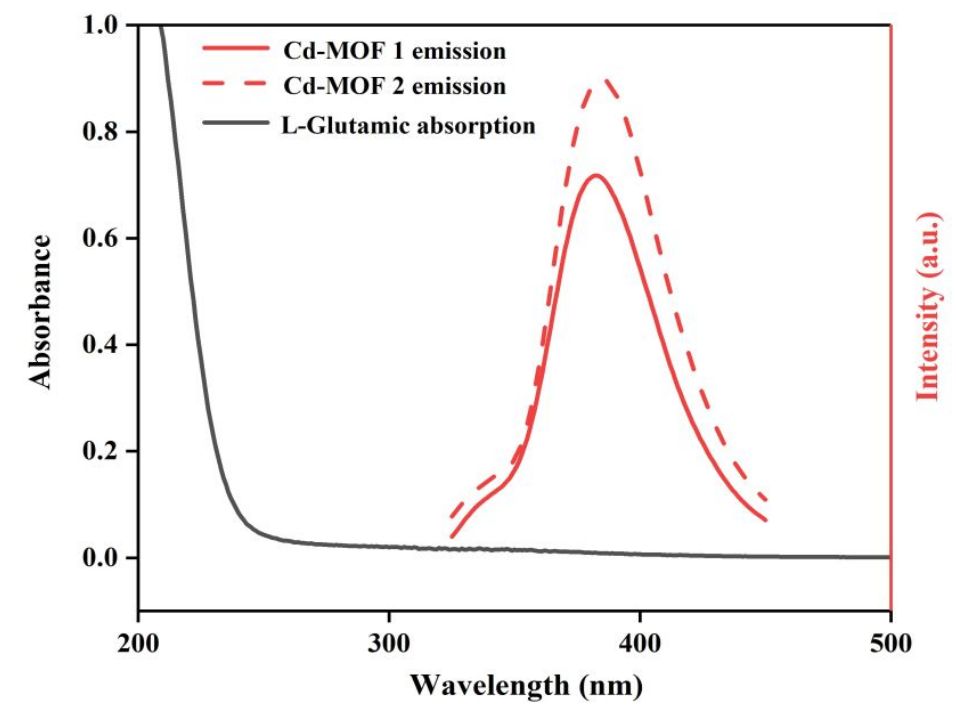

Figure S8. UV-vis spectrum of glutamate solution and luminescence spectrum of Cd-MOFs 1-2.

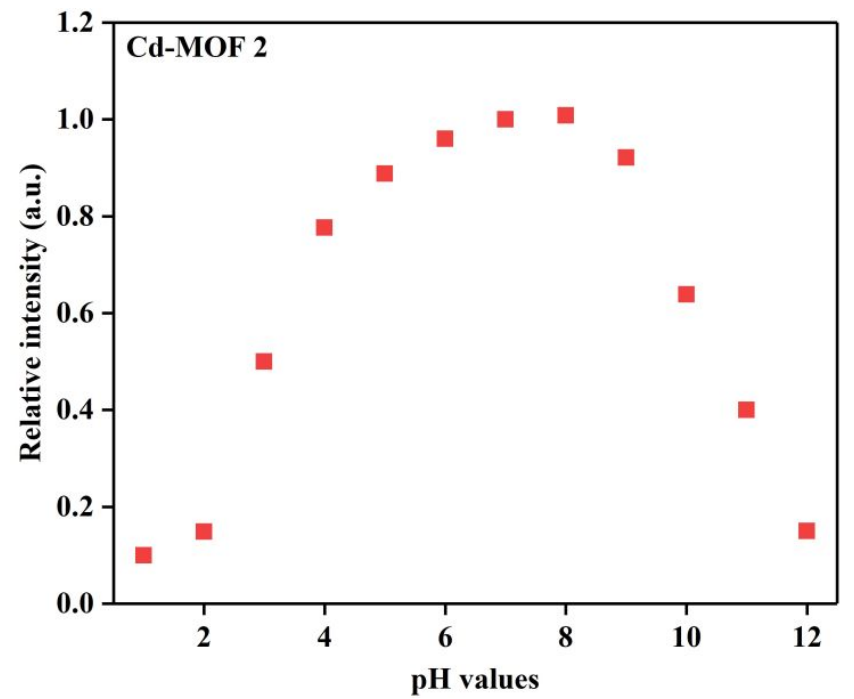

Figure S9. The scatter plot of the relationship between fluorescent intensity of Cd-MOF $\mathbf{2}$ and $\mathrm{pH}$ values.
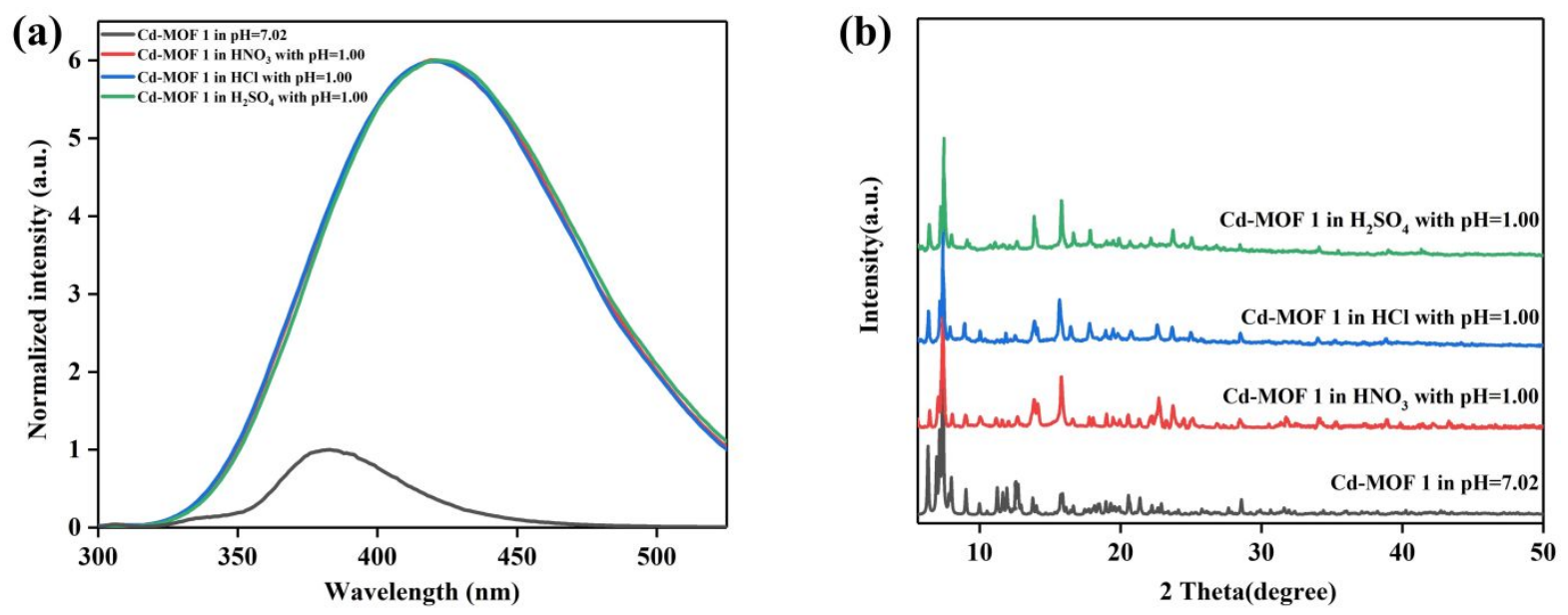

Figure S10. (a) The fluorescent intensity of 1 for different acids; (b) The PXRD patterns of 1 for different acids. 

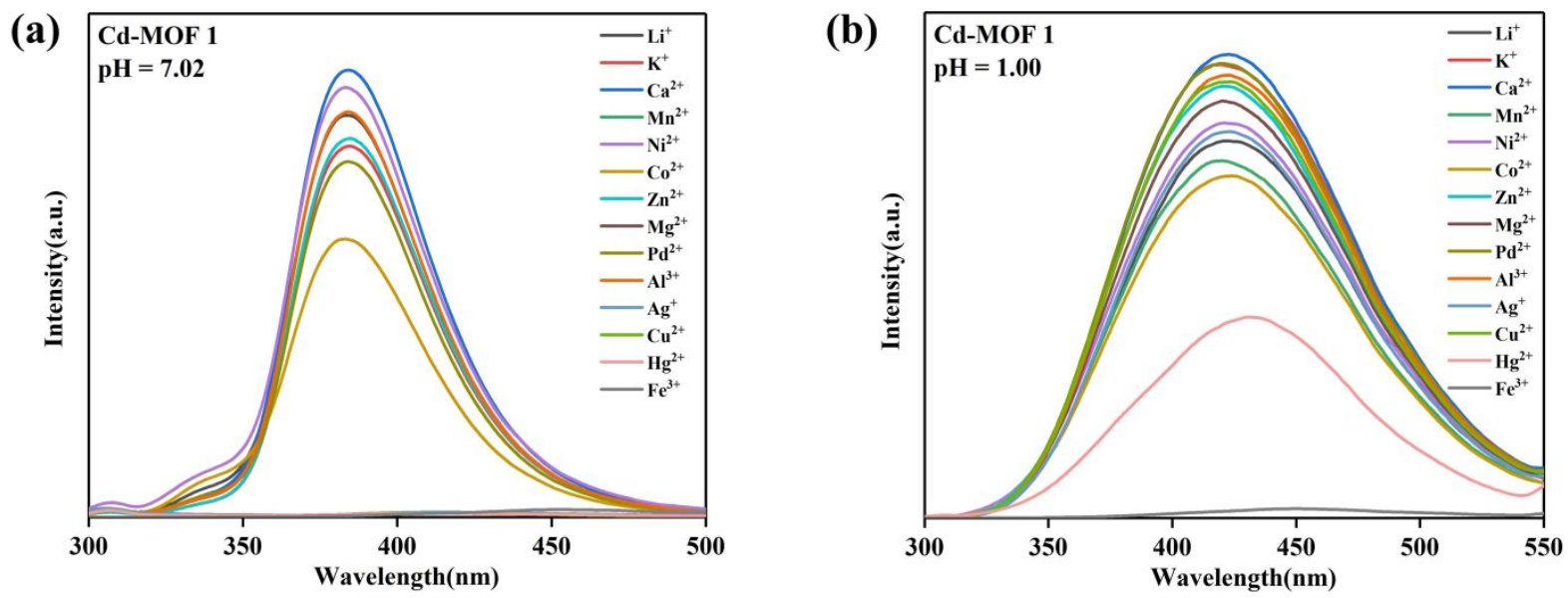

Figure S11. The fluorescent intensity of $\mathbf{1}$ for different metal ions in $\mathrm{pH}=1.00$ and 7.02.
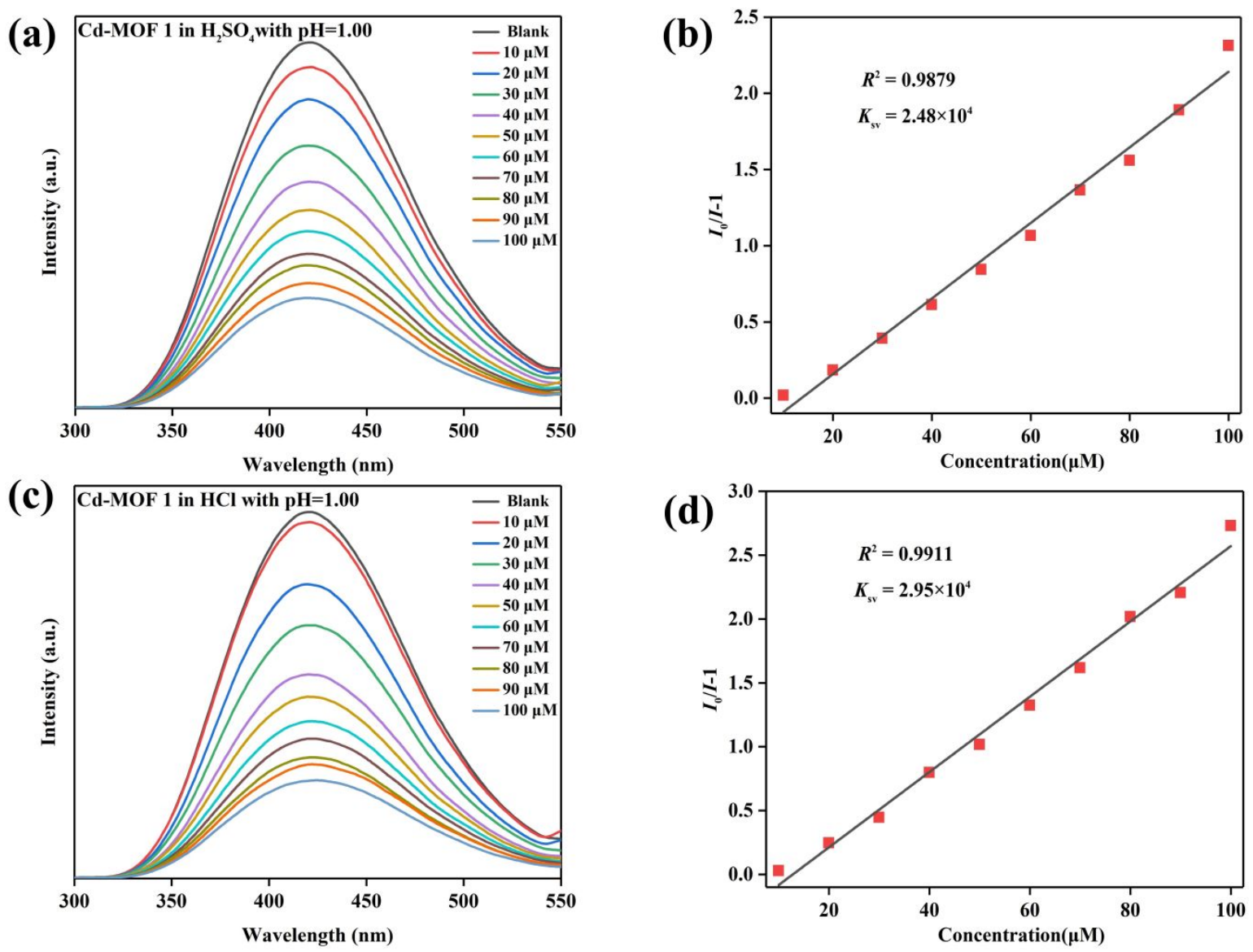

Figure S12. The fluorescent titration curves of 1 after the addition of different amounts of $\mathrm{Fe}^{3+}$ in (a) $\mathrm{H}_{2} \mathrm{SO}_{4}$; (c) $\mathrm{HCl}$; Linear responses of 1 to $\mathrm{Fe}^{3+}$ in (b) $\mathrm{H}_{2} \mathrm{SO}_{4}$; (d) $\mathrm{HCl}$. 


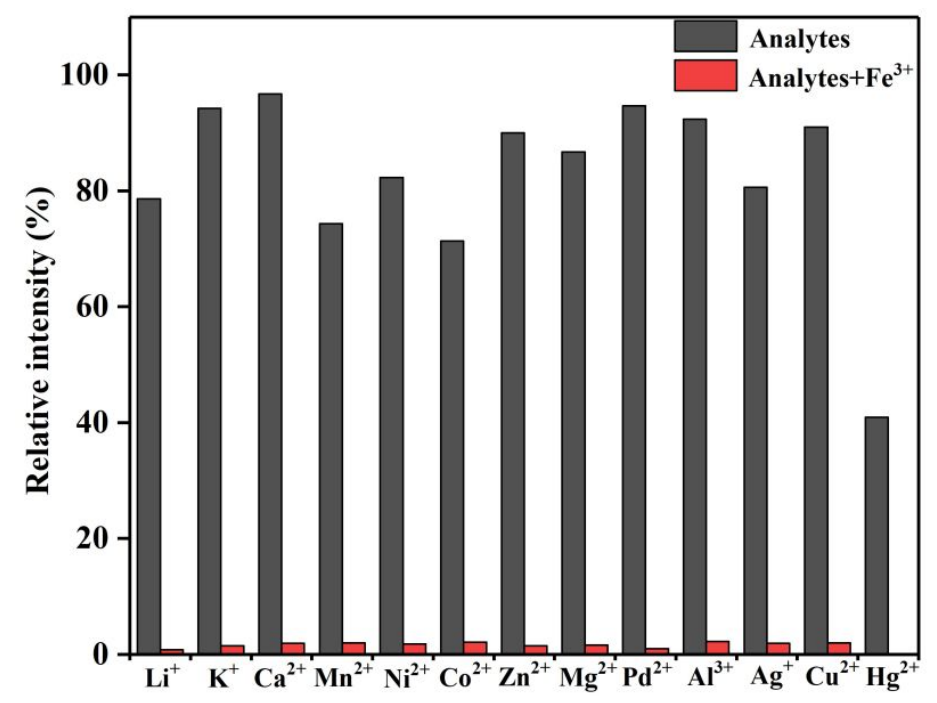

Figure S13. The relative luminescence intensities of 1 by different metal ions and selective detection of $\mathrm{Fe}^{3+}$ in $\mathrm{pH}=1.00$.
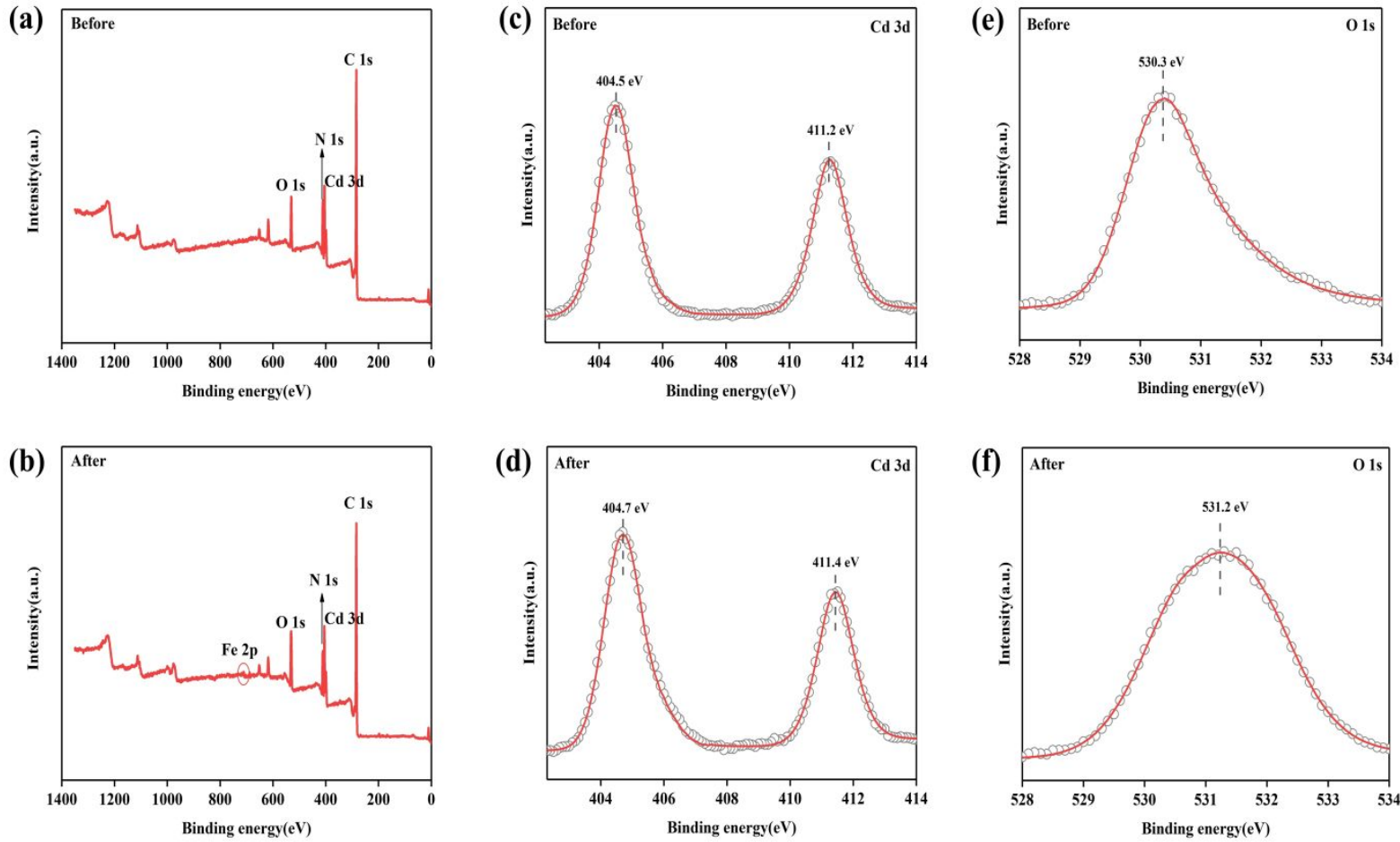

Figure S14. (a-b) XPS of 1 before and after soaked in $\mathrm{pH}=1.00$ solutions of $\mathrm{Fe}^{3+}$, (c-d) $\mathrm{Cd} 3 \mathrm{~d}$ XPS; (e-f) O 1s XPS before and after being soaked in $\mathrm{pH}=1.00$ solutions of $\mathrm{Fe}^{3+}$. 


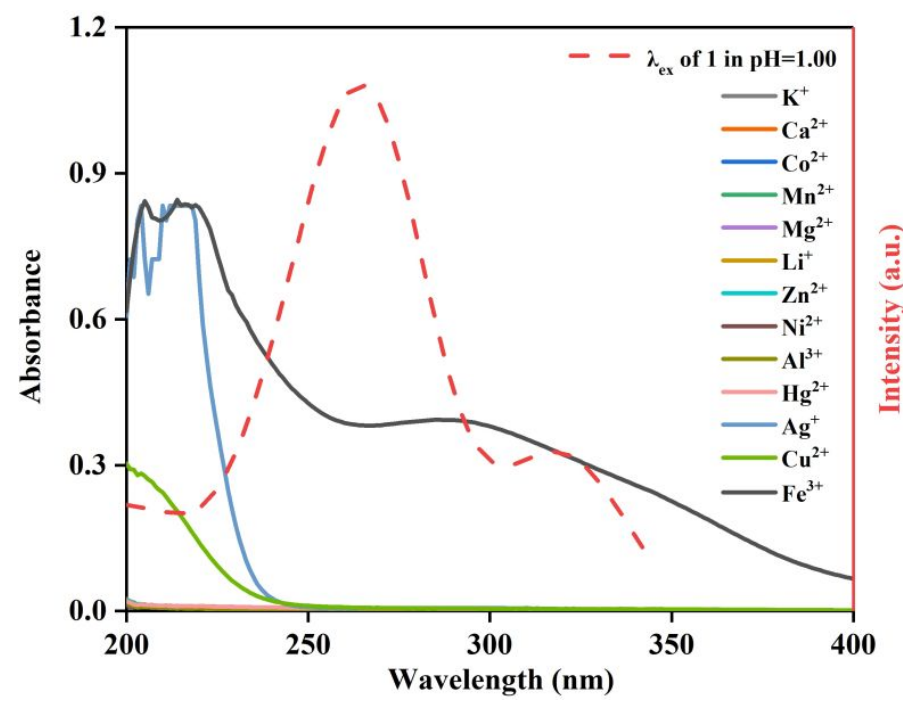

Figure S15. UV-vis spectra of cations in aqueous solutions and excitation spectra of Cd-MOF 1 in $\mathrm{pH}=1.00$.

Table S1 Selected bond lengths $(\AA)$ and angles $\left(^{\circ}\right)$ for Cd-MOFs 1-2.

\begin{tabular}{|c|c|c|c|}
\hline \multicolumn{4}{|c|}{ Cd-MOF 1} \\
\hline $\mathrm{Cd} 1-\mathrm{O} 1$ & $2.311(2)$ & $\mathrm{Cd} 1-\mathrm{N} 3$ & $2.382(3)$ \\
\hline $\mathrm{Cd} 1-\mathrm{O} 2$ & $2.603(3)$ & $\mathrm{Cd} 1-\mathrm{N} 2$ & $2.418(3)$ \\
\hline $\mathrm{Cd} 1-\mathrm{O} 4^{\mathrm{i}}$ & $2.340(3)$ & $\mathrm{Cd} 1-\mathrm{N} 6^{\mathrm{ii}}$ & $2.321(3)$ \\
\hline $\mathrm{Cd} 1-\mathrm{O}^{\mathrm{i}}$ & $2.581(3)$ & & \\
\hline $\mathrm{O} 1-\mathrm{Cd} 1-\mathrm{O} 2$ & $53.24(8)$ & $\mathrm{O} 1-\mathrm{Cd} 1-\mathrm{N} 3$ & $93.69(9)$ \\
\hline $\mathrm{O} 1-\mathrm{Cd} 1-\mathrm{O} 4 \mathrm{i}$ & $83.86(10)$ & $\mathrm{O} 1-\mathrm{Cd} 1-\mathrm{N} 2$ & $83.19(10)$ \\
\hline $\mathrm{O} 1-\mathrm{Cd} 1-\mathrm{O} 3 \mathrm{i}$ & $136.99(9)$ & $\mathrm{O} 1-\mathrm{Cd} 1-\mathrm{N} 6^{\mathrm{ii}}$ & $139.31(10)$ \\
\hline $\mathrm{N} 3-\mathrm{Cd} 1-\mathrm{O} 2$ & $98.95(10)$ & $\mathrm{N} 3-\mathrm{Cd} 1-\mathrm{N} 2$ & $171.32(10)$ \\
\hline $\mathrm{N} 3-\mathrm{Cd} 1-\mathrm{O} 3^{\mathrm{i}}$ & $87.92(10)$ & $\mathrm{O} 4^{\mathrm{i}}-\mathrm{Cd} 1-\mathrm{O} 3^{\mathrm{i}}$ & $53.12(9)$ \\
\hline $\mathrm{N} 2-\mathrm{Cd} 1-\mathrm{O} 2$ & $85.70(10)$ & $\mathrm{N} 66^{\mathrm{ii}}-\mathrm{Cd} 1-\mathrm{O} 2$ & $86.09(9)$ \\
\hline $\mathrm{N} 2-\mathrm{Cd} 1-\mathrm{O} 3^{\mathrm{i}}$ & $88.87(11)$ & $\mathrm{N} 6^{\mathrm{ii}}-\mathrm{Cd} 1-\mathrm{N} 3$ & $91.17(10)$ \\
\hline $\mathrm{O} 4-\mathrm{Cd} 1-\mathrm{O} 2$ & $136.25(9)$ & $\mathrm{N} 6^{\mathrm{ii}}-\mathrm{Cd} 1-\mathrm{N} 2$ & $96.48(10)$ \\
\hline $\mathrm{O} 4-\mathrm{Cd} 1-\mathrm{N} 3$ & $91.20(10)$ & $\mathrm{N} 6^{\mathrm{ii}}-\mathrm{Cd} 1-\mathrm{O} 4^{\mathrm{i}}$ & $136.44(11)$ \\
\hline $\mathrm{O} 4-\mathrm{Cd} 1-\mathrm{N} 2$ & 80.44 (10) & $\mathrm{N} 6^{\mathrm{ii}}-\mathrm{Cd} 1-\mathrm{O} 3^{\mathrm{i}}$ & $83.51(10)$ \\
\hline 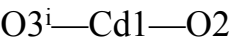 & $167.65(9)$ & & \\
\hline
\end{tabular}

Symmetry codes: (i) $x+1, y+1, z+1$; (ii) $x+1, y+1, z$.

Cd-MOF 2

$\begin{array}{llll}\mathrm{Cd} 1-\mathrm{O}^{\mathrm{i}} & 2.268(4) & \mathrm{Cd} 1-\mathrm{N} 4^{\mathrm{ii}} & 2.273(4) \\ \mathrm{Cd} 1-\mathrm{O} 2^{\mathrm{i}} & 2.424(4) & \mathrm{Cd} 1-\mathrm{N} 2 & 2.271(4)\end{array}$




\begin{tabular}{cccc}
$\mathrm{Cd} 1-\mathrm{O} 4$ & $2.194(4)$ & & \\
$\mathrm{O} 3^{\mathrm{i}}-\mathrm{Cd} 1-\mathrm{O} 2^{\mathrm{i}}$ & $55.78(14)$ & $\mathrm{O} 4-\mathrm{Cd} 1-\mathrm{O} 2^{\mathrm{i}}$ & $137.16(16)$ \\
$\mathrm{O} 3^{\mathrm{i}}-\mathrm{Cd} 1-\mathrm{N} 4^{\mathrm{ii}}$ & $139.57(17)$ & $\mathrm{O} 4-\mathrm{Cd} 1-\mathrm{N}^{\mathrm{ii}}$ & $111.75(17)$ \\
$\mathrm{O} 3^{\mathrm{i}}-\mathrm{Cd} 1-\mathrm{N} 2$ & $100.52(15)$ & $\mathrm{O} 4-\mathrm{Cd} 1-\mathrm{N} 2$ & $111.00(17)$ \\
$\mathrm{O} 3^{\mathrm{i}}-\mathrm{Cd} 1-\mathrm{C} 24^{\mathrm{i}}$ & $27.82(15)$ & $\mathrm{O} 4-\mathrm{Cd} 1-\mathrm{C} 24^{\mathrm{i}}$ & $116.94(17)$ \\
$\mathrm{O} 2^{\mathrm{i}}-\mathrm{Cd} 1-\mathrm{C} 24^{\mathrm{i}}$ & $28.05(15)$ & $\mathrm{N} 4^{\mathrm{ii}}-\mathrm{Cd} 1-\mathrm{O} 2^{\mathrm{i}}$ & $85.73(15)$ \\
\hline
\end{tabular}

Symmetry codes: (i) $x-1, y, z-1$; (ii) $x, y+1, z-1$.

Table S2 Summary of crystal data and structure refinement parameters of $\mathbf{1}$ and $\mathbf{1}$ in $\mathrm{pH}=1.00$

\begin{tabular}{|c|c|c|}
\hline & 1 & 1 in $\mathrm{pH}=1.00$ \\
\hline Crystal system & Triclinic & Triclinic \\
\hline Space group & $P \overline{1}$ & $P \overline{1}$ \\
\hline $\mathrm{a}(\AA)$ & $14.5402(14)$ & $14.4208(6)$ \\
\hline$b(\AA)$ & $15.0699(13)$ & $15.0139(6)$ \\
\hline c $(\AA)$ & $15.3074(15)$ & $15.2588(6)$ \\
\hline$\alpha\left(^{\circ}\right)$ & $96.787(3)$ & $96.806(1)$ \\
\hline$\beta\left(^{\circ}\right)$ & $105.180(3)$ & $105.303(1)$ \\
\hline$\gamma\left({ }^{\circ}\right)$ & $116.708(3)$ & $116.548(1)$ \\
\hline$V\left(\AA^{3}\right)$ & $2781.4(5)$ & 2741.45 (19) \\
\hline Z & 2 & 1 \\
\hline$D_{\text {calcd }}\left(\mathrm{Mg} \mathrm{m}^{-3}\right)$ & 1.067 & 1.444 \\
\hline$\mu\left(\mathrm{mm}^{-1}\right)$ & 0.43 & 0.46 \\
\hline$F(000)$ & 908 & 1226 \\
\hline$T(\mathrm{~K})$ & 170 & 170 \\
\hline$R_{\text {int }}$ & 0.080 & 0.078 \\
\hline Final $\mathrm{R}$ indices & $R_{1}=0.0510$ & $R_{1}=0.0665$ \\
\hline$[I>2 \operatorname{sigma}(\mathrm{I})]$ & $w R_{2}=0.1199$ & $w R_{2}=0.1473$ \\
\hline $\mathrm{R}$ indices (all data) & $R_{1}=0.0880$ & $R_{1}=0.1117$ \\
\hline & $w R_{2}=0.1033$ & $w R_{2}=0.1825$ \\
\hline Gof & 1.022 & 1.071 \\
\hline
\end{tabular}

Table S3 Selected bond lengths $(\AA)$ and angles $\left(^{\circ}\right)$ for $\mathbf{1}$ and $\mathbf{1}$ in $\mathrm{pH}=1.00$.

\begin{tabular}{ll|ll}
\hline & $\mathbf{1}$ & \multicolumn{2}{|c}{$1 \mathrm{in} \mathrm{pH}=1.00$} \\
\hline $\mathrm{Cd} 1-\mathrm{O} 1$ & $2.311(2)$ & $\mathrm{Cd} 1-\mathrm{O} 1$ & $2.303(4)$ \\
$\mathrm{Cd} 1-\mathrm{O} 2$ & $2.603(3)$ & $\mathrm{Cd} 1-\mathrm{O} 2$ & $2.588(4)$ \\
$\mathrm{Cd} 1-\mathrm{O} 4^{\mathrm{i}}$ & $2.340(3)$ & $\mathrm{Cd} 1-\mathrm{O} 4^{\mathrm{i}}$ & $2.335(4)$ \\
$\mathrm{Cd} 1-\mathrm{O} 3^{\mathrm{i}}$ & $2.581(3)$ & $\mathrm{Cd} 1-\mathrm{O} 5^{\mathrm{i}}$ & $2.559(5)$ \\
$\mathrm{Cd} 1-\mathrm{N} 3$ & $2.382(3)$ & $\mathrm{Cd} 1-\mathrm{N} 3$ & $2.364(4)$
\end{tabular}




\begin{tabular}{|c|c|c|c|}
\hline $\mathrm{Cd} 1-\mathrm{N} 2$ & $2.418(3)$ & $\mathrm{Cd} 1-\mathrm{N} 5$ & $2.406(5)$ \\
\hline $\mathrm{Cd} 1-\mathrm{N} 6^{\mathrm{ii}}$ & $2.321(3)$ & $\mathrm{Cd} 1-\mathrm{N} 2^{\mathrm{ii}}$ & $2.313(4)$ \\
\hline $\mathrm{O} 1-\mathrm{Cd} 1-\mathrm{O} 2$ & $53.24(8)$ & $\mathrm{O} 1-\mathrm{Cd} 1-\mathrm{O} 2$ & $53.20(13)$ \\
\hline $\mathrm{O} 1-\mathrm{Cd} 1-\mathrm{O} 4 \mathrm{i}$ & $83.86(10)$ & $\mathrm{O} 1-\mathrm{Cd} 1-\mathrm{O} 4^{\mathrm{i}}$ & $83.63(15)$ \\
\hline $\mathrm{O} 1-\mathrm{Cd} 1-\mathrm{O} 3 \mathrm{i}$ & $136.99(9)$ & $\mathrm{O} 1-\mathrm{Cd} 1-\mathrm{O} 5^{\mathrm{i}}$ & $136.86(14)$ \\
\hline $\mathrm{N} 3-\mathrm{Cd} 1-\mathrm{O} 2$ & $98.95(10)$ & $\mathrm{N} 3-\mathrm{Cd} 1-\mathrm{O} 2$ & $99.48(14)$ \\
\hline $\mathrm{N} 3-\mathrm{Cd} 1-\mathrm{O} 3^{\mathrm{i}}$ & $87.92(10)$ & $\mathrm{N} 3-\mathrm{Cd} 1-\mathrm{O} 5^{\mathrm{i}}$ & $87.38(15)$ \\
\hline $\mathrm{N} 2-\mathrm{Cd} 1-\mathrm{O} 2$ & $85.70(10)$ & $\mathrm{N} 5-\mathrm{Cd} 1-\mathrm{O} 2$ & $85.58(16)$ \\
\hline $\mathrm{N} 2-\mathrm{Cd} 1-\mathrm{O} 3^{\mathrm{i}}$ & $88.87(11)$ & $\mathrm{N} 5-\mathrm{Cd} 1-\mathrm{O} 5^{\mathrm{i}}$ & 88.97 (17) \\
\hline $\mathrm{O} 4-\mathrm{Cd} 1-\mathrm{O} 2$ & $136.25(9)$ & $\mathrm{O} 44^{\mathrm{i}}-\mathrm{Cd} 1-\mathrm{O} 2$ & $135.92(14)$ \\
\hline $\mathrm{O} 4-\mathrm{Cd} 1-\mathrm{N} 3$ & $91.20(10)$ & $\mathrm{O} 4-\mathrm{Cd} 1-\mathrm{N} 3$ & $91.17(14)$ \\
\hline $\mathrm{O} 4-\mathrm{Cd} 1-\mathrm{N} 2$ & $80.44(10)$ & $\mathrm{O} 4-\mathrm{Cd} 1-\mathrm{N} 5$ & $80.19(16)$ \\
\hline $\mathrm{O} 3-\mathrm{Cd} 1-\mathrm{O} 2$ & $167.65(9)$ & $\mathrm{O} 5^{\mathrm{i}}-\mathrm{Cd} 1-\mathrm{O} 2$ & $167.80(14)$ \\
\hline $\mathrm{O} 1-\mathrm{Cd} 1-\mathrm{N} 3$ & $93.69(9)$ & $\mathrm{O} 1-\mathrm{Cd} 1-\mathrm{N} 3$ & $94.11(15)$ \\
\hline $\mathrm{O} 1-\mathrm{Cd} 1-\mathrm{N} 2$ & $83.19(10)$ & $\mathrm{O} 1-\mathrm{Cd} 1-\mathrm{N} 5$ & $83.06(16)$ \\
\hline $\mathrm{O} 1-\mathrm{Cd} 1-\mathrm{N} 6^{\mathrm{ii}}$ & $139.31(10)$ & $\mathrm{O} 1-\mathrm{Cd} 1-\mathrm{N} 2^{\mathrm{ii}}$ & $139.22(14)$ \\
\hline $\mathrm{N} 3-\mathrm{Cd} 1-\mathrm{N} 2$ & $171.32(10)$ & $\mathrm{N} 3-\mathrm{Cd} 1-\mathrm{N} 5$ & $171.15(15)$ \\
\hline $\mathrm{O} 4^{\mathrm{i}}-\mathrm{Cd} 1-\mathrm{O} 3^{\mathrm{i}}$ & $53.12(9)$ & $\mathrm{O} 4^{\mathrm{i}}-\mathrm{Cd} 1-\mathrm{O} 5^{\mathrm{i}}$ & $53.23(14)$ \\
\hline $\mathrm{N} 66^{\mathrm{ii}}-\mathrm{Cd} 1-\mathrm{O} 2$ & $86.09(9)$ & $\mathrm{N} 2{ }^{\mathrm{ii}}-\mathrm{Cd} 1-\mathrm{O} 2$ & $86.06(14)$ \\
\hline $\mathrm{N} 66^{\mathrm{ii}}-\mathrm{Cd} 1-\mathrm{N} 3$ & $91.17(10)$ & $\mathrm{N} 2{ }^{\mathrm{ii}}-\mathrm{Cd} 1-\mathrm{N} 3$ & $90.95(15)$ \\
\hline $\mathrm{N} 66^{\mathrm{ii}}-\mathrm{Cd} 1-\mathrm{N} 2$ & $96.48(10)$ & $\mathrm{N} 2^{\mathrm{ii}}-\mathrm{Cd} 1-\mathrm{N} 5$ & $96.67(16)$ \\
\hline $\mathrm{N} 6^{\mathrm{ii}}-\mathrm{Cd} 1-\mathrm{O} 4^{\mathrm{i}}$ & $136.44(11)$ & $\mathrm{N} 2{ }^{\mathrm{ii}}-\mathrm{Cd} 1-\mathrm{O} 4^{\mathrm{i}}$ & $136.74(16)$ \\
\hline $\mathrm{N} 6^{\mathrm{ii}}-\mathrm{Cd} 1-\mathrm{O} 3^{\mathrm{i}}$ & $83.51(10)$ & $\mathrm{N} 2^{\mathrm{ii}}-\mathrm{Cd} 1-\mathrm{O} 5^{\mathrm{i}}$ & $83.74(15)$ \\
\hline
\end{tabular}

Symmetry codes: (i) $x+1, y+1, z+1$; (ii) $x+1, y+1, z$.

Table S4 Average fluorescence lifetime of double exponential fit.

\begin{tabular}{|c|c|c|c|c|c|c|}
\hline & $\mathrm{A}_{1}$ & $\tau_{1}(\mathrm{~ns})$ & $\mathrm{A}_{2}$ & $\tau_{2}(\mathrm{~ns})$ & $\mathrm{R}^{2}$ & $\tau_{\mathrm{avg}}(\mathrm{ns})$ \\
\hline 1 in $\mathrm{pH}=1.00$ & 3102.37 & $\begin{array}{c}1.20 \\
65.9 \%\end{array}$ & 262.14 & 7.36 & 0.9935 & 3.30 \\
\hline 1 in $\mathrm{pH}=7.02$ & 2211.85 & $\begin{array}{c}1.17 \\
37.5 \%\end{array}$ & 992.05 & 4.34 & 0.9976 & 3.15 \\
\hline 1 in $1 \mathrm{mM} \mathrm{Fe}^{3+}$ & 3375.55 & $\begin{array}{c}1.42 \\
81 \%\end{array}$ & 113.01 & 9.57 & 0.9850 & 2.92 \\
\hline
\end{tabular}

$\tau_{\mathrm{avg}}=\left(\mathrm{A}_{1} \tau_{1}^{2}+\mathrm{A}_{2} \tau_{2}^{2}\right) /\left(\mathrm{A}_{1} \tau_{1}+\mathrm{A}_{2} \tau_{2}\right)$ 


\section{References}

[1] Sheldrick, G. M. SHELXTL NT, version 5.1, program for solution and refinement of crystal structures, University of GÖttingen, GÖttingen, Germany, 1997.

[2] Bruker 2000, SMART, version 5.0, SAINT-plus version 6, SHELXTL, version 6.1, and SADABS version 2.03, Bruker AXS Inc., Madison, WI.

[3] Spek, A. L. Implemented as the PLATON Procedure, a Multipurpose Crystal lographic Tool, Utrecht University, Utrecht, The Netherlands, 1998.

[4] Zong, Z. A.; Bi, C. F.; Zhu, Z.; Fan, C. B.; Meng, X. M.; Zhang, X.; Fan, Y. H. Structure, sensing and photocatalytic properties of two multifunctional 3D luminescent coordination polymers based on an N-heterocyclic carboxylic acid. New J. Chem. 2018, 42, 8905-8913.

[5] Van Der Sluis, P.; Spek, A. L. BYPASS: an effective method for the refinement of crystal structures containing disordered solvent regions, Acta Crystallographica Section A 1990, 46, 194-201.

[6] Spek, A. Single-crystal structure validation with the program PLATON, Journal of Applied Crystallography 2003, 36, 7-13.

[7] Liu, Z. Q.; Zhao, Y.; Wang, P.; Kang, Y. S.; Azam, M.; Al-Resayes, S. I.; Liu, X. H.; Lu, Q. Y.; Sun, W. Y. Fluorescent sensing and selective adsorption properties of metal-organic frameworks with mixed tricarboxylate and 1H-imidazol-4-yl-containing ligands. Dalton Trans. 2017, 46, 9022-9029.

[8] Wang, F.; Ke, X.; Zhao, J.; Deng, K.; Leng, X.; Tian, Z.; Wen, L.; Li, D. Six new metal-organic frameworks with multi-carboxylic acids and imidazole-based spacers: syntheses, structures and properties. Dalton Trans. 2011, 40, 11856-11865. 